\title{
Strong IT Effort Underpins MOE/MAR Success
}

$\overline{\text { Penny Hackenbrook-Rogers, Trevor Godfrey, David Eagan, Monique Pitre and Anna Barbosa }}$

\section{Introduction}

The implementation of a Medication Order Entry/Medication Administrative Record (MOE/MAR) system inevitably involves substantial technological and change-process challenges. No system can meet the needs of all potential user groups, nor can any hospital's information technology (IT) budget for a MOE/ MAR-type initiative be unlimited. This paper describes the process by which the University Health Network's (UHN) IT department, called Shared Information Management Services (SIMS), in implementing this new system attempted to take advantage of recent technological advances, satisfy users and do so within budget constraints. The challenges of doing so in this large, academic hospital organization are identified, drawing on both our successful and less successful design and implementation efforts.

Prior to the deployment of MOE/MAR, the hospital information system (HIS) was being used by clinicians and nonclinicians throughout UHN as part of their daily workflow to help with a wide variety of care processes, including

- Patient registration and admission

- Order entry for laboratory investigation, diagnostic imaging, diet and other ancillary services

- Results review for diagnostic tests (including lab and diagnostic imaging)
- Managing the storage and retrieval of medical images (PACS)

- Documenting admission assessments, allergy assessments and other patient information

- Review of clinical encounter documentation including admission and discharge summaries, surgical procedures, ambulatory visits and numerous other encounter notes

- Information processing and storage for many departments/ labs

The other major component of the UHN IT infrastructure essential to the new MOE/MAR environment was the Pharmacy Information System (Rx System). That system is used by pharmacists to manage UHN's medication inventory. Prior to MOE/MAR, pharmacists entered medication orders into the $\mathrm{Rx}$ System in response to paper-based orders received from physicians.

\section{Approach and Model}

Considerable additional customization had to be added to UHN's HIS and Rx System environment to support the on-line order entry and administration management for medications. These additional capabilities required SIMS to

- develop procedures to allow existing paper-based workflows to be mapped into an on-line environment 
- build a custom database of medication procedures based on UHN's formulary of standard medications

- develop multiple HL7 -based interfaces between the HIS and Rx System (Health Level Seven 2006)

- develop user interface ordering screens customized for each hospital service

- integrate a third-party medication interaction checking product

- implement an on-line equivalent for the Medication Administration Record (MAR)

- develop reports required by clinicians to manage the medication order/administration process

- implement a point-of-care device strategy involving personal computers (PCs), laptops and mobile carts

\section{Choosing the Right Systems Model}

One of the most significant decisions UHN had to make was choosing the best approach for the underlying systems architecture. Three possible options were considered:

1. Use the existing HIS (but add medication inventory management capability).

2. Use the existing Rx System (but add on-line medication order entry capability).

3. Integrate the HIS and Rx System.

SIMS chose the multi-system approach (see Figure 1), since no one system alone could address all the business and clinical requirements.

However, the two systems had different architectures, which created many interfacing challenges for SIMS, including negoti-

Figure 1. Multi-system approach
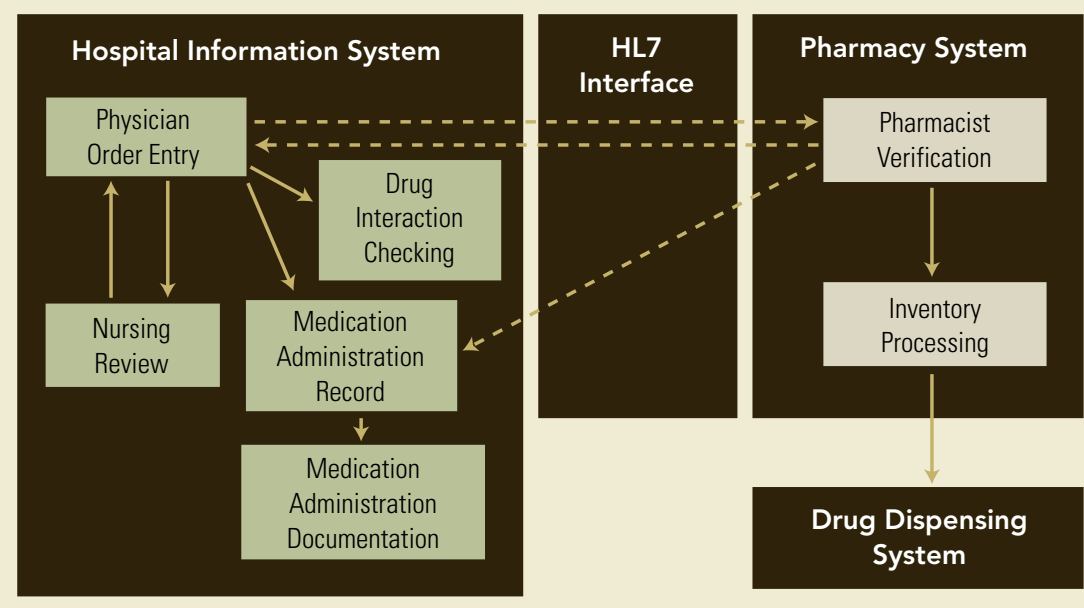

ating with both vendors to determine which system would be modified to accommodate workflow requirements.

\section{System Interfaces}

The effort required to integrate the HIS and Rx System centred largely on developing new interfaces between the two systems. It was equally important to assess the potential impact that implementing MOE/MAR might have on any existing interfaces. A rigorous change management process was also implemented to support the ongoing need for interface changes.

\section{Application Development and Maintenance}

UHN undertook a pilot launch of MOE/MAR in February 2003. The main objective of this pilot project was to identify any functionality gaps between the users and the application to support medication order entry and medication administration.

Beyond the pilot, UHN took a phased approach to implementing MOE/MAR to the remaining Nursing Units. This was necessary given the size, complexity and geographic dispersion of UHN's clinical programs. There were eight groups of Nursing Units or clusters to implement across the campuses, encompassing seven clinical programs with more than 20 service groupings. With each new cluster, new workflow requirements were identified to meet the specific needs of each of the clinical services. Building on the technical requirements of previous clusters, SIMS project and technical teams incrementally incorporated new application customization into the MOE/MAR environment. This required a high degree of adaptability and flexibility with our system-build process to accommodate changes.

For small to mid-size institutions contemplating MOE/ MAR initiatives, we encourage them to consider whether they could, instead, accommodate a "big bang" approach rather than the slower, incremental approach taken at UHN. These organizations might assess their ability to conduct the analysis across a representative selection of the units first so they can build a complete and robust endstate system from the start.

\section{User-Interface Customization} A substantial part of the IT effort involved mapping approximately 2,000 medications from UHN's formulary into the HIS medication database and building 10,000 predefined "typical orders" based on various dosage, route and frequency combinations for each of those medications. From these typical 
Table 1. System interfaces

\begin{tabular}{|l|l|}
\hline $\begin{array}{l}\text { Bi-directional orders } \\
\text { interface }\end{array}$ & $\begin{array}{l}\text { The outbound order interface carries } \\
\text { new, changed and discontinued } \\
\text { orders from the HIS to the Rx System. } \\
\text { Pharmacists review these orders, } \\
\text { and any medication product changes } \\
\text { made on the Rx System are sent back } \\
\text { to the HIS over the inbound order } \\
\text { interface. The interface keeps both } \\
\text { systems synchronized with respect to } \\
\text { the orders and medication products. }\end{array}$ \\
\hline Master file update & $\begin{array}{l}\text { The Rx System controls which } \\
\text { medication products are available } \\
\text { to be ordered. A real-time, one-way } \\
\text { interface between the Rx System } \\
\text { and the HIS is used for loading } \\
\text { and changing medication product } \\
\text { information in the HIS database. }\end{array}$ \\
\hline
\end{tabular}

orders, a custom "order set," or collection of the most commonly used orders, was created for each hospital service, totalling more than 130 . These order sets were presented to physicians, giving them simple and easy-to-use "point \& click" order entry capability. This not only saved physicians time and reduced the chance for error, it also helped UHN enforce standards and promote best practices through the use of approved medication orders appropriate to a particular service.

The challenges associated with creating custom order sets revealed the unique complexities of standardizing medication orders in a large, complex academic setting (e.g., new processes and medications are frequently introduced throughout UHN).

Figure 2. Standard "common medication" on-line order screen

\begin{tabular}{|c|c|c|}
\hline \multicolumn{3}{|l|}{ GiM Medications Options } \\
\hline Meds on Paper MAR & $\square$ (Gravol) 25-50mg N/PO q4h pm & $\square$ Metoprolol \\
\hline Analgesics & $\square$ (Stemetil) 5.10mg I MPPO q6h pm & $\square$ Nitroglycerin \\
\hline$\square$ Acetsminophen & Antilinfectives & $\square$ (PIswix) Clopidogrel Bisulfate \\
\hline$\square$ Acetaminophen $3256-650 \mathrm{mg}$ PO $\mathrm{g} 4 \mathrm{~h} \mathrm{pm}$ & $\square$ Ampictilin & $\square$ RAMlipril \\
\hline Acetaminophen $500-1000 \mathrm{mg}$ PO a $6 \mathrm{~h} \mathrm{prn}$ & $\square$ Azithromycin & $\square$ Spironolactone \\
\hline$\square$ (Dilaudid) Hydromorphone & $\square$ Cefazolin Sodium & CNS Agents \\
\hline$\square$ Morphine Sulfate & $\square$ Ceftriaxone Sodium & $\square$ Diazepam \\
\hline Morphine Sultate Drip & $\square$ cipronoxacin (PO) & $\square$ Halopendol \\
\hline$\square$ (Tyenol *3) Acetamin wicodeine $30 \mathrm{mg}$ & $\square$ Moxinoxacin & $\square$ LorAZapam \\
\hline Anticosgulants \& Antiplatelets & $\square$ Metronidazole & $\square$ LorAZepam 0.5-1. Omg POrsL an \\
\hline (Aspirin) Acetsis alicylic Acid & $\square$ Vancomycin $\mathrm{HCl}$ & $\square$ Risperidone \\
\hline$\square$ (Aspirin) ECASA B1 mg PO daily & Cardiovascular & Diabetichnsulins \\
\hline Enoxaparin Sodium & $\square$ (Aggrenox) Dipyridamole ER \& ASA NF & Humalog \\
\hline$\square$ Heparin & $\square$ AmLoDipine Besylate & $\square$ Humulin $\mathrm{N}$ \\
\hline Tinzaparin Sodium & $\square$ Atenolal & $\square$ Humulin R \\
\hline$\square$ Wartarin & $\square$ Atonrastatin & $\square$ Norolin ge $30 / 70$ \\
\hline W Warfarin Daily Order & $\square$ Dittiazem & Diabeticoral Hypoglycemics \\
\hline Antiemetics & $\square$ Enalapril & \\
\hline$\square$ (Gravol) DimenhyDRINATE & $\square$ HYdrochloroTHlazide & $\square$ metrormin $\mathrm{HCl}$ \\
\hline
\end{tabular}

In some instances, UHN decided to standardize across the entire organization, such as adopting the use of generic names rather than trade names for all medication products in the UHN formulary. In other cases, medication orders were standardized just within a service, such as General Internal Medicine or General Surgery, so that the Pick List for all physicians within that service would be the same.

\section{Quality Assurance}

Each of the nearly 2,000 medications and 10,000 typical orders had to be tested to ensure that they were clinically correct. Due to the complexity and sheer volume of the typical orders, it took nearly nine months to build and test the entire formulary. This testing was done by pharmacists.

Nurses were also integrally involved in the quality assurance process. The nurses reviewed the order sets and nursing functions within the system to determine how the order was displayed, whether the order information was presented in a logical manner and if the information appearing on the MAR, Medication Summary, 7-Day Medication History and other reports was accurate. A special testing lab equipped with groups of computers physically clustered together was assembled where pharmacists and nurses could work side by side with SIMS staff for quality assurance testing processes.

Typically, physicians were active contributors to the creation and review of the custom order sets for their own particular services. The SIMS project and technical teams learned that having all the clinical groups involved in the Quality Assurance process was the best way to ensure that the procedures, typical orders and workflow around them met the professional standards of the three groups, were consistent with UHN policies and were clinically correct and appropriate.

The continuous cycle of creating, refining and testing standards for typical orders and order sets during the project had to be built into the overall project plan and schedule. Because of the phased approach it was often necessary to go back to incorporate change requests from earlier implementations.

\section{Clinical Decision Support}

UHN undertook the MOE/MAR project to take advantage of the clinical decision support capabilities that electronic medication management and the electronic patient record offered for reducing patient risk due to medication error.

The MOE/MAR solution was designed to automatically check for medication-medication and medication-allergy incompatibilities as well as for duplicate medication orders 
Figure 3. Standardized on-line order set screen (clinical program-specific)

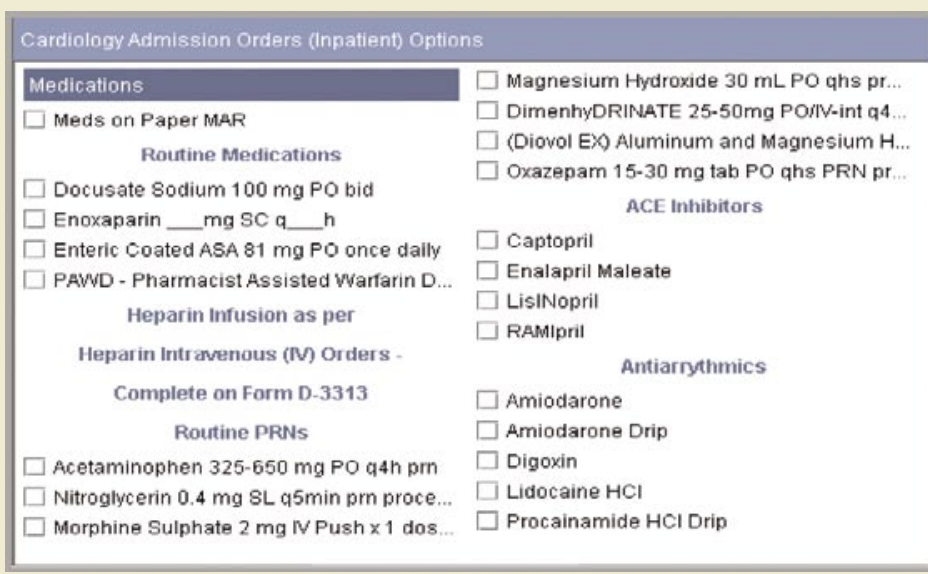

incomplete. For instance, several of the product files were in fact based on U.S. information (e.g., pharmaceutical naming) and incompatible with Canadian norms. The HIS vendor continues to work with the third-party decision support vendor to remedy this situation.

\section{Device/Delivery Model}

A key benefit of the MOE/MAR project was to move the "point of access" to information closer to the "point of care," specifically by providing clinicians computerized access to information at patients' bedsides. It was intended that this could improve patient safety by facilitating the real-time verification of patient information before medications were administered, and supporting nurses' ability to document the administration of those medications immediately thereafter.

Achieving this required SIMS to develop and execute a hardware device strategy encomand orders that are inconsistent with lab results. For anyone ordering medications (i.e., physicians, nurse practitioners), the operational concept behind the MOE/MAR clinical decision support was to alert them to any potential problems at point of order entry. This was accomplished using third-party medication interaction checking software completely integrated within the HIS application as well as within the Rx System.

At the project outset, when the medication checking alerts were being configured, it was decided that the decision support function should function in an "overly safe" manner. It was soon discovered, however, that this third-party product was overly cautious - according to physicians who believed that far too many alerts were issued. Consequently, the order-alerting mechanism was "dialled back" so that only the most critical alerts would be triggered. Physicians needed time to get used to the overall system first. Pharmacists were also provided with clinical alerts at the time of order verification on the Rx System. However, the alert level on this system was left high, as there is established tolerance by pharmacists for the alert interruption.

Additional alerts were eventually reintroduced into the system, and more specific requirements were provided to the vendor in order to make the alerting procedure "smarter." Since the original implementation of the third-party software, UHN has also begun to implement its own medication interaction checking outside of the third-party database using available HIS functionality.

One of the final decision support lessons learned by UHN was a "translation" issue unique to Canadian hospitals. UHN implemented a system provided by a U.S. vendor. Despite the vendor's assurances that the product had been modified for the Canadian market, these modifications were later found to be passing the following challenges:

- How many and what type of computers would be needed by clinicians?

- Where should the equipment be located to optimally deliver information quickly and easily?

- How could the effects of potential system downtimes be mitigated?

- What mobility requirements needed to be addressed to ensure access to information at the right place and time?

\section{Wireless Device Strategy Supports Mobility Requirements}

Resolution of the mobility question was especially critical to UHN implementing an effective MOE/MAR solution. Previously, stationary PCs were located in all Nursing Units to support the range of HIS capabilities being used by the clinicians. However, physicians, nurses and pharmacists were recognized as highly mobile staff. MOE/MAR was the first project within UHN's overall Electronic Patient Record initiative that highlighted the hospital's need to add a mobility/wireless capability so that clinicians could interact with the computer close to the patients.

A dedicated technical sub-team was assigned to focus on implementing a wireless device strategy. They conducted a series of trials to assess the requirements of UHN's wireless infrastructure, including the number and locations of access points needed and the most appropriate physical devices to be deployed. In addition to providing adequate coverage for initial MOE/MAR implementations, this sub-team also determined that the wireless 
infrastructure would have to be scalable (in order to support a growing number of devices as the EPR initiative continued to unfold). It also needed to be "multi-generational" so it could be easily upgraded over time without having to be replaced.

A key aspect of the wireless device strategy centred on exploring the range of mobile carts that could be used by clinicians to get information and medications to the bedside. A manufacturer provided several prototype carts with fixed wireless laptops for trial to support the functions being performed by the users. These included nursing carts with drawers for medications, and physician rounding carts. The trials showed that, although relatively inexpensive, the initial carts were too big and cumbersome to be moved easily in and out of patient rooms.

While no single cart was considered "ideal," the carts that the device team ultimately deployed worked well. Specifically, UHN chose to use thin-client devices as the mobile PCs in order to reduce cart size and weight and to optimize power consumption so that the rechargeable batteries supplying power to the carts would last for an entire shift. The decision to use thin clients, which depend on a server-based computing model for access to applications, fit well into UHN's existing IT infrastructure strategy, where Citrix had already been deployed to optimize application delivery through "virtualization." This would also

Figure 4. Mobile Point-of-Care (POC) device

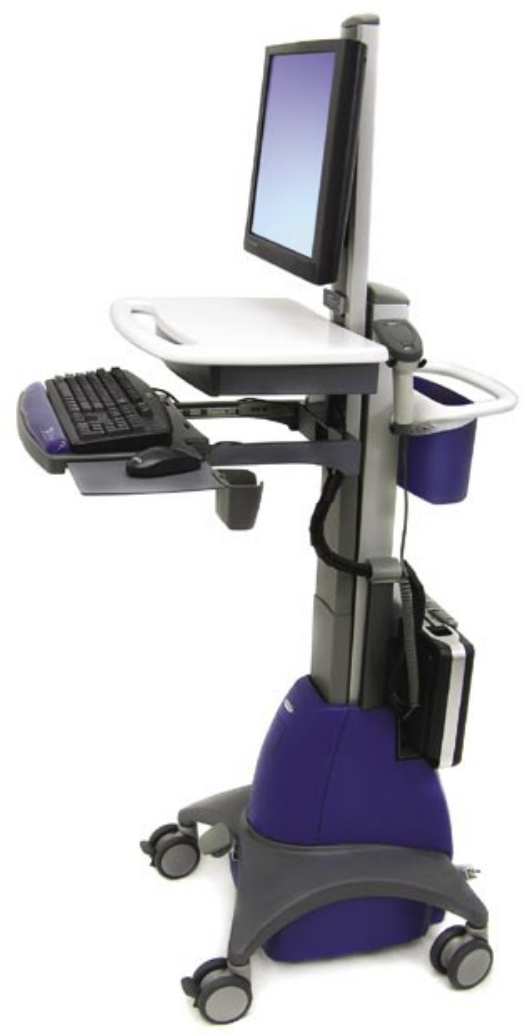

simplify ongoing application and PC management. SIMS continues to assess new and improved cart designs, considering new requirements that continue to emerge, such as barcode scanning functionality.

\section{Device Strategy Addresses Downtime Scenario}

SIMS implemented a simple and effective solution to the eventuality of MOE/MAR downtime. Every 30 minutes, outstanding medication orders for all patients on a given ward would be downloaded to a PC (with natively attached printer) installed on the unit specifically for this purpose. The PC and printer were to be plugged into the hospital's emergency power system, which has generator backup to protect against possible power outage. If there is a system outage nurses can sign onto the downtime PC and print the Downtime Report containing the MAR information they need.

\section{Reports}

Many different reports were developed by SIMS for the new MOE/MAR environment. Several reports are launched as HTML pages within the HIS while others are available as printed reports. Some examples of MOE/MAR reports are shown in Table 3.

Table 2. Example of deployment in a GIM Unit

\begin{tabular}{|l|c|c|}
\hline \multicolumn{3}{|c|}{ General Internal Medicine Unit } \\
\hline Device Type & $\begin{array}{c}\text { Quantity Pre- } \\
\text { MOE/MAR }\end{array}$ & $\begin{array}{c}\text { Quantity Post- } \\
\text { MOE/MAR }\end{array}$ \\
\hline Nursing station desktops & 10 & 10 \\
\hline Laptop & 1 & 1 \\
\hline Reports printer & 1 & 1 \\
\hline Blood label printer & 1 & 1 \\
\hline Mobile cart & 0 & 6 \\
\hline Med room device & 0 & 2 \\
\hline Rounding cart & 0 & 1 \\
\hline Isolation room & 0 & 5 \\
\hline Whiteboard & 1 & 1 \\
\hline Downtime PC & 0 & 1 \\
\hline Total devices on unit & $\mathbf{1 4}$ & \\
\hline
\end{tabular}

Source: Shared Information Management Services 
The Medications Preparation Report in fact filled a preexisting gap in the HIS functionality. Prior to MOE/MAR, there was no function to indicate to nurses exactly which medications needed to be obtained from the medication dispensing machines located in each ward. This ensures that nurses prepare the right medications for the right patient.

The 7-Day Medication History Report arose from the need for nurses to see what medications their patients had recently been given. The new electronic MAR only displays medication orders that still need to be given but not those previously administered. The 7-Day Medication History Report provides this "historical" information, saving clinicians from having to perform individual chart reviews to obtain previous medication administration data.

The Inpatient Whiteboard shows a list of all the patients on the ward, with corresponding indicators denoting activities that need to be undertaken for each (e.g., lab tests, radiology tests, medication administration). The Inpatient Whiteboard Report gives nurses a quick, visual indication of patient-specific tasks that need to be completed without requiring them to log onto the system.

Table 3. Examples of MOE/MAR reports

\begin{tabular}{|l|l|}
\hline On-line HTML Reports & Hardcopy Reports \\
\hline Medications Preparation Report & $\begin{array}{l}\text { Active Medications Transfer } \\
\text { Report }\end{array}$ \\
\hline 7-Day Medication History Report & Missed Dosage Report \\
\hline Inpatient Whiteboard Report & Untimely Documentation Report \\
\hline
\end{tabular}

Figure 5. UHN inpatient order whiteboard

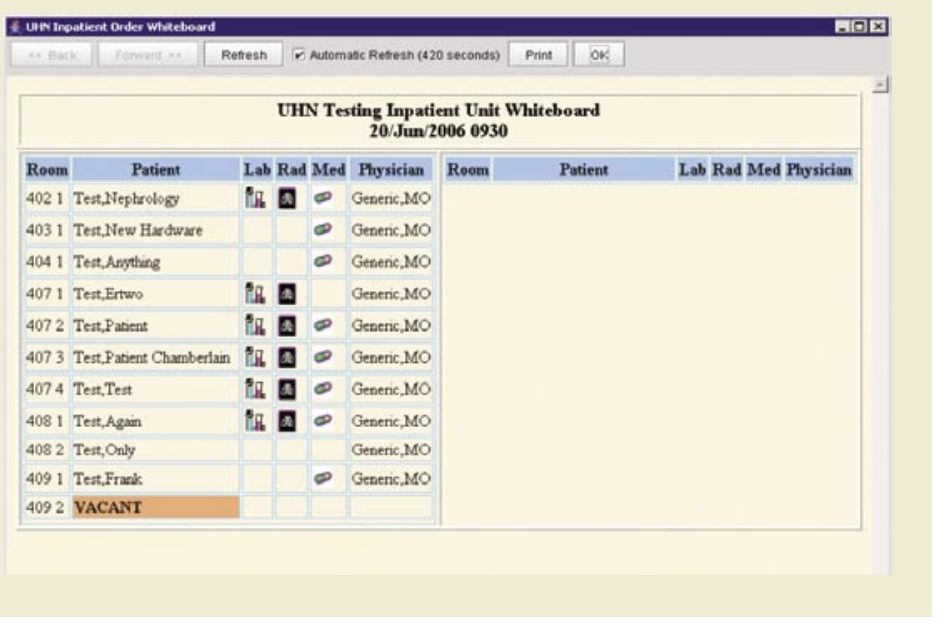

Table 4. Technical challenges and considerations matrix

\begin{tabular}{|c|c|}
\hline Technical Challenges & Technical Consideration Factors \\
\hline System architecture & $\begin{array}{l}\text { - Enterprise vs. interfaced systems } \\
\text { approach }\end{array}$ \\
\hline System interfaces & $\begin{array}{l}\text { - Impact to existing interfaces (i.e., } \\
\text { ADT) } \\
\text { - Mapping between interfaced } \\
\text { systems (i.e., routes, frequencies, } \\
\text { products) }\end{array}$ \\
\hline $\begin{array}{l}\text { Application development \& } \\
\text { maintenance }\end{array}$ & $\begin{array}{l}\text { - Trial with a production pilot } \\
\text { - Customize for usability; } \\
\text { standardize for best practice } \\
\text { - Get clinical engagement in quality } \\
\text { assurance }\end{array}$ \\
\hline Clinical decision support & $\begin{array}{l}\text { - Set the alert levels to avoid alert } \\
\text { fatigue }\end{array}$ \\
\hline Device delivery model & $\begin{array}{l}\text { - Computers/devices } \\
\text { - number, type, location, mobility } \\
\text { requirements } \\
\text { - System downtime planning for } \\
\text { business continuity }\end{array}$ \\
\hline Reports & $\begin{array}{l}\text { - Address functionality gaps with } \\
\text { customized report views } \\
\text { - Audit reports to measure system } \\
\text { use compliance }\end{array}$ \\
\hline
\end{tabular}

The Active Medications Transfer Report provides hardcopy MAR information to nurses in non-MOE/MAR areas when a patient is transferred from a MOE/MAR Unit to a non-MOE/ MAR Unit (to date, MOE/MAR is under development in Intensive Care Units and Transplant Units). Other reports, such as the Missed Dosage Report and the Untimely Documentation Report, were developed to provide Nursing management with information they needed to manage their staff's medication administration activities.

In the early days of the project, considerable time and effort was spent in unnecessary, overly iterative development of these reports. This resulted in early versions of reports that were serving too many purposes or were unusable because they were too large to run in a timely manner. The report development process did improve with careful clarification, up front, on report scope, purpose and recipients. 


\section{Lessons Learned}

1. Where possible, choose a single-system approach to on-line medication management; avoid the need for complex interfaces and systems integration.

2. If a multi-system model is adopted, recognize the impact that operational processes may have on the interfaces between those systems.

3. Do not underestimate the time, effort and resources required to properly manage third-party product vendors.

4. System interfaces can be designed to support workflow that may be missing from the individual systems themselves.

5. Small to mid-sized hospitals should consider analyzing all representative and key workflows up front so that a complete endstate system can be built from the start.

6. Encourage clinical stakeholders to agree to basic system design and build standards, including medication management and clinical workflow policies, before designing the system.

7. Test systems in a real production environment before going live to ensure they will stand up to user traffic load and handle all normal and anomalous workflow situations.

8. Solicit input, review and approval from all three clinical disciplines when building custom medication orders sets; expect standards for medication orders and medication procedures to evolve over the course of the project.

9. Ensure that system design and clinical workflow can easily be changed to accommodate changes to the medication formulary - new medications being added, old medications being retired, etc.

10. When implementing clinical decision support alerts, start with only the most important ones and then add more over time as clinician comfort level increases.

11. Recognize that U.S. designed systems, despite vendor assurances, may need additional modifications to work effectively in a Canadian setting.

12. A wireless, mobile device strategy that gets the point of information access as close as possible to the Point of Care is key to a successful MOE/MAR implementation.

13. All spending decisions for mobile carts must also involve considerations of future technological advances.

14. Ensure clinicians still have access to critical medication management information in the event of a system downtime scenario.

\section{Conclusion}

From a technical perspective, implementing MOE/MAR at UHN tested our mettle. Many unique technical and vendor management issues were uncovered related to integrating two disparate systems. Understanding and building to the clinical requirements of diverse, multidisciplinary groups became an exercise in negotiations to translate medication management standards and operational practices into a functional and userfriendly on-line system. Ensuring that appropriate wireless devices and supporting infrastructure could support complex workflows required its own strategy in parallel with MOE/ MAR. Finally, new reports needed to be developed to support a smooth transition to electronic MOE/MAR functionality from the previous paper-based environment.

In spite of these obstacles, MOE/MAR is now "live" in more than 25 Inpatient Units across the UHN hospital campuses, including the Emergency departments, pre-admission clinics and same-day service areas. As a result, UHN now has a robust and scalable technical architecture upon which to build more intelligent clinical alerts, and to continue with additional initiatives to completely replace paper-based patient charts with Electronic Patient Records (EPR).

\section{References}

Health Level Seven Inc. 2006. About HL7. Retrieved October 1, 2006. <http://www.hl7.org/about/hl7about.htm\#WhatisHL7>.

\section{About the Authors \\ Penny Hackenbrook-Rogers is Manager of the Patient Care Management Systems of Shared Information Management Services (SIMS) at University Health Network. Contact: penny. hackenbrook-rogers@uhn.on.ca}

Trevor Godfrey, RN, BA, PMP, is a Project Manager with the Patient Care Management Systems of Shared Information Management Services (SIMS) at University Health Network.

David Eagan is the Service Support Manager, Information Technology with Shared Information Management Services (SIMS) at University Health Network.

Monique Pitre, BSc (Pharm), is Manager, Pharmacy Clinical Informatics, and the Infectious Disease Pharmacist, University Health Network.

Anna Barbosa, is a Senior Application Specialist with the Pharmacy Clinical Informatics department at University Health Network.

\section{Commentary \\ Lessons Are Worth Heeding for Any Facility}

\section{Denni McColm}

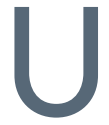
$\mathrm{HN}$ is to be applauded for its successful initiative with MOE/MAR. Its efforts have saved lives, and will continue to prevent adverse drug events.

From our experiences with a comparable but much smaller implementation of computerized physician order entry and the electronic MAR, we perceive many similarities. The lessons learned are worth heeding for any facility planning a similar implementation.

Citizens Memorial Healthcare (CMH) is a rural healthcare network located in Southwest Missouri. The network includes a 74-bed rural hospital, five long-term care facilities, 16 physi- 
cian clinics and a home care agency. All patient care services are connected into one electronic medical record (EMR). Network physicians enter their own orders for procedures and medications, eliminating paper medical records. The initiative, known as Project Infocare, serves 1,600 users. well.

The following lessons learned at UHN ring true for us as

If possible choose a single-system approach to on-line medication management; avoid the need for complex interfaces and systems integration. Fortunately, we were creating all new systems when we implemented computerized physician order entry and the electronic MAR at CMH. Because we chose an integrated strategy, we did not face the daunting task of interfacing systems as complex as those required by nurses, physicians and pharmacists to order, dispense and administer medications.

Small to mid-sized hospitals should consider analyzing all representative and key workflows up front so that a complete end-state system can be built from the start. We also experienced a need to "rebuild" the pharmacy and provider order entry tables so that the system was win-win for both the pharmacists (who tend to see medications as inventory items) and physicians (who tend to see medications in doses). Our system provides the capability to bridge the two viewpoints, but forethought, planning and communication are required to make it a reality.

Solicit input, review and approval from all three clinical disciplines when building custom medication orders sets; expect standards for medication orders and medication procedures to evolve over the course of the project. Like UHN, we built our own ordering conventions for medications. Building these "order strings," as we identified them, from the historical medication orders throughout our network proved useful. Vendors now have pre-set ordering conventions for purchase that will simplify this process for facilities in the future.

When implementing clinical decision support alerts, start with only the most important ones and then add more over time as clinician comfort level increases. We also implemented clinical alerts originally in an "overly safe" mode. We quickly realized that we were inducing "alert fatigue," as it is now called in the industry. Like UHN, we backed off the severe alerting status. In the future, I see clinical alerting becoming more sophisticated. For one thing, we will become better at identifying specific allergies. Currently, if our nurses enter an allergy to a combination drug that includes acetaminophen, the physician is flagged to the acetaminophen allergy - even though the allergy may have been to the other medication in the combination drug. Training in allergy specificity will help with this problem. Third-party vendors will also improve over time in their ability to alert appropriately.

Our congratulations to UHN on their successful implementation of a complex system across so many care units!

\section{About the Author}

Denni McColm is the $\mathrm{ClO}$ of Citizens Memorial Healthcare in Bolivar, Missouri - the first-ever non-academic and rural hospital to win the coveted Davies Organizational Award.

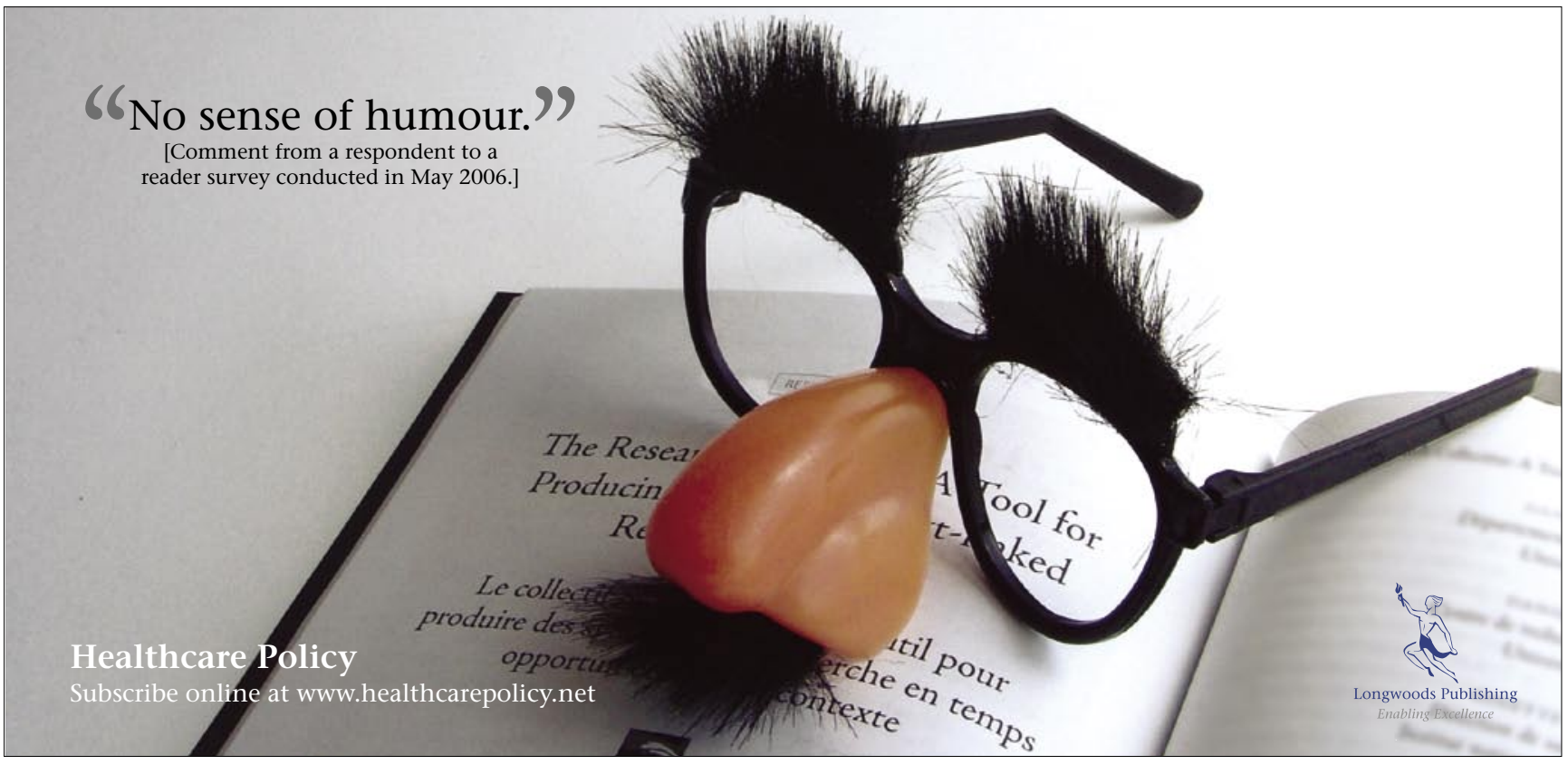

